\title{
Shrinkage Effect on Cracking Resistance of Flexural Reinforced Concrete Members
}

\author{
Robertas BALEVIČIUS*, Mindaugas AUGONIS**, Rẻda BISTRICKAITË***, \\ Saulius DILIŪNAS $* * * *$ \\ *Vilnius Gediminas Technical University Sauletekio ave.11,10223 Vilnius, Lithuania, E-mail: robertas.balevicius@vgtu.lt \\ **Kaunas University of Technology, Studentu st.48, 51367 Kaunas, Lithuania, E-mail: Mindaugas.augonis@ktu.lt \\ ***Kaunas University of Technology, Studentu st. 48, 51367 Kaunas, Lithuania, E-mail: reda.bistrickaite@ktu.lt \\ ****Kaunas University of Technology, Studentu st. 56, 51424 Kaunas, Lithuania, E-mail: saulius.diliunas@ktu.lt \\ cross ref http://dx.doi.org/10.5755/j01.mech.24.1.17901
}

\section{Introduction}

The shrinkage strains developing in concrete with time before loading is one of factor which can affect the cracking resistance of RC members under the of short- or sustained flexure. Depending on environmental conditions the early age concrete shrinkage can produce a significant tensile stress in concrete and compressive stress in reinforcement bars.

The shrinkage stresses are caused by the restrained effect produced by reinforcement bars in order to maintain compatibility of concrete and bar strains along with the selfbalanced equilibrium conditions. Due to this, the shrinkageinduced stresses are undergone with tensile creep strains. In this case, the tensile creep causes the rate-dependent evolution of stresses, requiring the solution of Volterra integrodifferential equation of second kind [1]. So, in calculation of the cracking moment of the cross-section the shrinkage induced stresses should be accounted for. As it has been recently demonstrated by Bischoff and Scanlon [2,3], the evaluation of reduction in the moment of cracking due to restrained shrinkage evolved prior loading extremely improves the theoretical prediction of deflections for the GFRP RC cracked cross-sections.

As a rule, the shrinkage along with creep strains is taken into account for the long-term analysis of the crosssection. Actually, this is a rare case for the short-term deformational analysis of the elements. It is obvious that evolution of retrained shrinkage is dependent on the bond properties between the surrounding concrete and reinforcement bars. In fact, for the perfect bond properties developed at early age before loading, the transfer of tensile load (caused by the restrained shrinkage) to the surrounding concrete highly increases. This is the case to account for the timeevolution of bond stresses along with the restrained shrinkage for the improved prediction of cracking resistance of RC cross-sections.

In practice, the restrained shrinkage evolution can be reduced using special types of mixes allowing for diminishing amount of cement paste. It significantly affects the shrinkage strain evolution in time, but some initial tensile stress in concrete remains anyway.

For the symmetrically reinforced members, the retrained shrinkage induced tensile stress in concrete is not complicated task, when the tensile creep induced stress rate evolution in time is neglected. However, the effect is this rate significantly affects the time-dependent stress-strain state of the member. More difficult predictive calculations are provided for the unsymmetrically reinforced members, when the restrained shrinkage strains should be accounted for both cross-section edges requiring the development of the set of recurrent algebraic equations that allows avoiding solution Volterra equations using relaxation functions.

In the present work, the numerical and analytical models for predicting the cracking resistance parameters are developed on the basis of the above mentioned peculiarities.

\section{A numerical model}

Let us consider a doubly reinforced, uncracked and statically determinate element of arbitrary cross-section with a vertical axis of symmetry. Let a reference level of this axis be selected at the top fibre of the cross-section and pointed downward. So, the vertical coordinate $y$ remains positive over the entire height of the cross-section. Suppose there are no loads imposed, but the element is undergone a shortening, i.e., the stress-strain state is resulted from the action of displacement induced by a free shrinkage strain uniformly distributed over the cross-section.

Moreover, assume that the stress in compression, causing the shortening of the element, be positive. Thereby, the free-shrinkage strain is positive provided. Meanwhile, the negative values of stresses and strains are attributed to tension. Due to bond between the embedded bars and surrounding concrete, the bars restrict the element shortening and transfer a portion of tensile stress to the surrounding concrete. Since the properties of concrete are age-dependent, the transferred stress varies with time, but in a different manner that the shrinkage strain evolves with time [4]. For the case of non-symmetrically reinforced concrete element, the restrained shrinkage causes a time-dependent bending of the member with the gradual evolution of the curvature in time. For symmetrical arrangement of the bars, there is no bending of the element.

Suppose a linear distribution for the stress and strain at any point $y$ below the top fibre of the cross-section at time $t$, then,

$$
\sigma(y, t)=\sigma_{c}^{t o p}(t)+\frac{y}{h}\left[\sigma_{c}^{b o t}(t)-\sigma_{c}^{t o p}(t)\right],
$$

and, similarly,

$$
\varepsilon(y, t)=\varepsilon_{c}^{t o p}\left(t, t_{0}\right)+\frac{y}{h}\left[\varepsilon_{c}^{b o t}\left(t, t_{0}\right)-\varepsilon_{c}^{t o p}\left(t, t_{0}\right)\right],
$$


where: $\sigma_{c}^{t o p}(t), \sigma_{c}^{b o t}(t)$ and $\varepsilon_{c}^{t o p}\left(t, t_{0}\right), \varepsilon_{c}^{b o t}\left(t, t_{0}\right)$ are the stresses and strains in the top and bottom fibres of the crosssection at time $t, t_{0}$ is the age of loading, i.e., time of initiation of autogenous shrinkage strain, $h$ is the height of the cross-section.

The self-balanced equilibrium conditions at time $t$ can be written in this way:

$$
\left\{\begin{array}{l}
\sum H=0, \int_{A} \sigma(y, t) d A+\sigma_{s}(t) A_{s}+\sigma_{s 1}(t) A_{s 1}=0 \\
\sum M^{t o p}=0, \int_{A}+\sigma_{s 1}(t) A_{s 1} a_{1}=0
\end{array},\right.
$$

where: $\sigma_{s}(t)=E_{s} \varepsilon(h-a, t), \sigma_{s 1}(t)=E_{s} \varepsilon\left(a_{1}, t\right)$ are the stress of reinforcements coming from the conditions of compatibility $\varepsilon_{s}(t)=\varepsilon(h-a, t), \varepsilon_{s 1}(t)=\varepsilon\left(a_{1}, t\right)$ and Hooke's law, $A_{s}$ and $A_{s 1}$ are the reinforcement, located near the bottom and top fibres of the cross-section, respectively, areas, $a$ and $a_{1}$ are the distances from the bottom and top fibres of the cross-section to the center of reinforcement $A_{s}$ and $A_{s 1}$, respectively, $E_{s}$ is the modulus of elasticity of the reinforcement bars, $A$ is the area of concrete cross-section.

Following the principle of superposition $[5,6]$, the total strain of concrete at time $t$ is composed of the elastic, tensile creep and free shrinkage strains and is expressed as follows:

$$
\begin{aligned}
& \varepsilon_{c}\left(t, t_{0}\right)=\sigma_{c}\left(t_{0}\right) J\left(t, t_{0}\right)+\int J(t, \tau) d \sigma_{c}(\tau)+ \\
& +\varepsilon_{s h r}\left(t, t_{s}\right),
\end{aligned}
$$

where: $J(t, \tau)=\frac{1}{E_{c}(\tau)}+C(t, \tau)$ is the creep compliance resulted from a unit stress of concrete, $E(\tau)$ is the modulus of elasticity and specific creep of concrete at age $\tau, t_{s} \equiv t_{0}$ is the time, at which the autogenous shrinkage strain starts developing.

Elimination of the integrals in the relationship (4) results in the following formulae:

$$
\varepsilon_{c}\left(t, t_{0}\right)=\frac{\sigma_{c}(t)}{E_{c}(t)}+\sigma_{\xi}\left(t, t_{0}\right) C^{*}\left(t, t_{0}\right)+\varepsilon_{s h r}\left(t, t_{s}\right),
$$

where:

$$
C^{*}\left(t, t_{0}\right)=\frac{1}{E_{c}\left(t_{0}\right)}-\frac{1}{E_{c}(t)}+C\left(t, t_{0}\right)
$$

is the pure specific creep function, involving the partly recovered instantaneous strain, and

$$
\sigma_{\xi}\left(t, t_{0}\right)=-C^{*}\left(t, t_{0}\right)^{-1} \int_{t_{0}}^{t} \sigma_{c}(\tau) \frac{\partial}{\partial \tau} J(t, \tau) \mathrm{d} \tau
$$

is an average stress of concrete in a sense to fulfill Volterra's integral term in $\left[t, t_{0}\right]$.

Thus, from equation (5), it can be find the stress at the top and the bottom fibres at time $t$ as follows:

$$
\begin{aligned}
& \sigma_{c}^{t o p}(t)=E_{c}(t)\left[\begin{array}{l}
\varepsilon_{c}^{t o p}(t)-\sigma_{\xi}^{t o p}\left(t, t_{0}\right) C^{*}\left(t, t_{0}\right)- \\
-\varepsilon_{s h r}\left(t, t_{s}\right)
\end{array}\right], \\
& \sigma_{c}^{b o t}(t)=E_{c}(t)\left[\begin{array}{l}
\varepsilon_{c}^{b o t}(t)-\sigma_{\xi}^{b o t}\left(t, t_{0}\right) C^{*}\left(t, t_{0}\right)- \\
-\varepsilon_{s h r}\left(t, t_{s}\right)
\end{array}\right] .
\end{aligned}
$$

Application of the above formulae to solve explicitly the set of equations (3) is impossible due to required integration of age-varying creep and instantaneous-elastic strain functions. In this case, the theoretically exact solution can only be given numerically, when the recurrent algebraic equations are substituted for the integral equations. To this end, let us use a discretized time scale $\left(t_{0}, t_{1}, \ldots, t_{i-1}\right.$, $\left.t_{i}, \ldots, t_{n}\right)$ and consider all the stress increments in the previous intervals to get the acting stress at time of consideration $t_{n}$ (where $n$ is the number of the final time being considered). Then, an average stress of concrete in a sense to fulfill Volterra's integral term in $\left[t_{n}, t_{0}\right]$ can be written:

$$
\begin{aligned}
\sigma_{\xi}^{t o p}\left(t_{n}, t_{0}\right) & =\frac{\sum_{i=1}^{i=n-1} \sigma_{\xi}^{t o p}\left(t_{i}, t_{i-1}\right)\left[J\left(t_{n}, t_{i-1}\right)-J\left(t_{n}, t_{i}\right)\right]+\sigma_{\xi}^{t o p}\left(t_{n}, t_{n-1}\right) C^{*}\left(t_{n}, t_{n-1}\right)}{C^{*}\left(t_{n}, t_{0}\right)}, \\
\sigma_{\xi}^{b o t}\left(t_{n}, t_{0}\right) & =\frac{\sum_{i=1}^{i=n-1} \sigma_{\xi}^{b o t}\left(t_{i}, t_{i-1}\right)\left[J\left(t_{n}, t_{i-1}\right)-J\left(t_{n}, t_{i}\right)\right]+\sigma_{\xi}^{b o t}\left(t_{n}, t_{n-1}\right) C^{*}\left(t_{n}, t_{n-1}\right)}{C^{*}\left(t_{n}, t_{0}\right)} .
\end{aligned}
$$

Here, an average value of the stress, $\sigma_{\xi}\left(t_{n}, t_{0}\right)$ (for $n=1,2, \ldots, n=n$ ) fulfilling Volterra's integral term within the entire time interval $t_{n}-t_{0}$ is linked to that stress in the discrete time interval $\left[t_{i-1}, t_{i}\right]$ by its middle value, such that $\sigma_{\xi}\left(t_{i}, t_{i-1}\right)=\sigma_{c}\left(t_{i}\right) / 2+\sigma_{c}\left(t_{i-1}\right) / 2$, since $t_{i}-t_{i-1} \rightarrow 0$ is being supposed.

Finally, by substituting $\sigma_{\xi}^{\text {top }}\left(t_{n}, t_{0}\right)$ and $\sigma_{\xi}^{b o t}\left(t_{n}, t_{0}\right)$ for $\sigma_{\xi}^{t o p}\left(t, t_{0}\right)$ and $\sigma_{\xi}^{b o t}\left(t, t_{0}\right)$ in Eq. (8) and (9) and inserting the obtained formulae into the system (3), (for $n=1,2, \ldots, n=n$ ), we operate with a recurrent set of linear algebraic equations with respect to unknown strains $\varepsilon_{c}^{\text {top }}\left(t_{n}, t_{0}\right), \varepsilon_{c}^{\text {bot }}\left(t_{n}, t_{0}\right)$ over all $t_{0}, t_{1}, \ldots, t_{i-1}, t_{i}, \ldots, t_{n}$, in $t_{n^{-}}$ $t_{0}$. Solution of these equations now is a simple task along with a single-integration over the coordinate $y$. It was conveniently performed adopting matrix calculus. For the prolonged time of consideration required, if the time step is held constant, the solution is hardly applicable due to wasting a lot of CPU time and computer memory. Hence, to avoid 
these difficulties and achieving a desirable computational performance, the numerical analysis was carried out by using an increasing time step, $\Delta t_{i}=\sqrt[16]{10} \Delta t_{i-1}$.

When the stress functions are resolved, the coefficient of aging, induced by the restrained shrinkage, was simply calculated numerically by the following expression:

$$
\chi\left(t_{n}, t_{0}\right)=\left[\begin{array}{l}
\frac{\sigma_{\xi}\left(t_{n}, t_{0}\right) k\left(t_{n}\right)}{\sigma_{c}\left(t_{n}\right)-\sigma_{c}\left(t_{0}\right)}+ \\
+\frac{\sigma_{c}\left(t_{n}\right)\left(1-k\left(t_{n}\right)\right)-\sigma_{c}\left(t_{0}\right)}{\sigma_{c}\left(t_{n}\right)-\sigma_{c}\left(t_{0}\right)}
\end{array}\right],
$$

where: $k\left(t_{n}\right)=\frac{C^{*}\left(t_{n}, t_{0}\right)}{C\left(t_{n}, t_{0}\right)}$ is the factor accounting for the influence of partially recoverable instantaneous strain on the coefficient of ageing.

\section{Results}

Let us analyze the evolution of stress-strain state with time of a singly reinforced concrete element due to

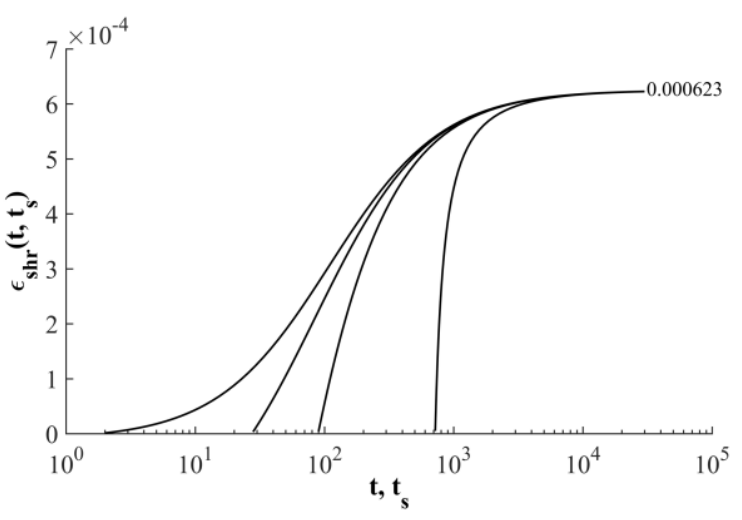

a)

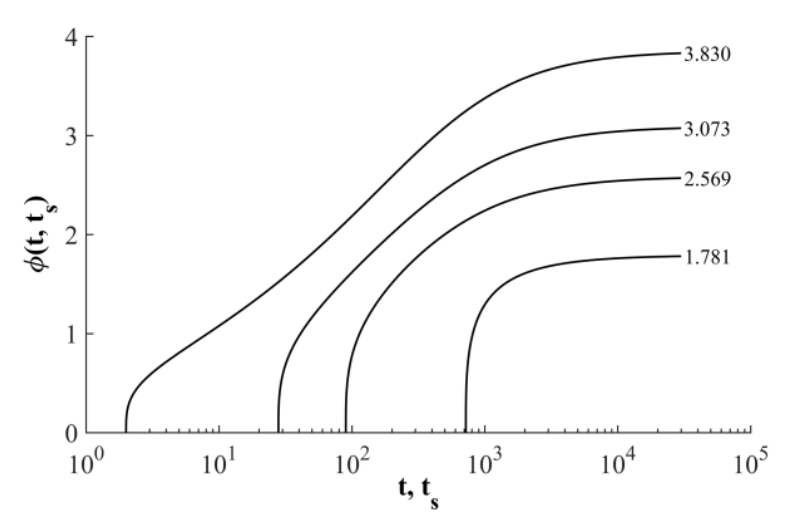

c) shrinkage. Two cross-sections having the same dimensions of $h=0.6 \mathrm{~m}$ and $b=0.3 \mathrm{~m}$, but of different reinforcement areas, namely, $A_{s}=18.60 \cdot 10^{-4} \mathrm{~m}^{2}$ and $A_{s}=37.20 \cdot 10^{-4} \mathrm{~m}^{2}$, located at $a=0.050 \mathrm{~m}$ from its centre to the bottom of the beam, are considered. The creep and shrinkage properties have been calculated by formulae given in the code EC-2 (EN 1992-1-1 2004) [7]. The elasticity modulus at the age of loading was also defined using code EC-2 relationship, $E_{c}\left(t_{0}\right)=\sqrt{\beta_{c c}\left(t_{0}\right)} E_{c}(28) \quad-\quad$ (where Young modulus $E_{c}(28)=2.15 \cdot 10^{4} \sqrt[3]{f_{c m} / 10}$, for $f_{c m}=f_{c k}+8 ; f_{c k}$ is the characteristic value of the compressive strength of the cylinders). The factor $\beta_{c c}(t)$ evaluates the age of the concrete (EN 1992-1-1 2004). To fulfil the theory of creep, the values of $\phi\left(t, t_{0}\right)$, determined by code (EN 1992-1-1 2004), should be additionally multiplied by factor $\beta_{c c}(t)$ due to code-based methodological peculiarities (see remarks in [8]). A rapid hardening cement type, effective thickness of the element equal $h_{0}=200 \mathrm{~mm}$ and relative humidity $R H$ of $60 \%$ have been also selected.

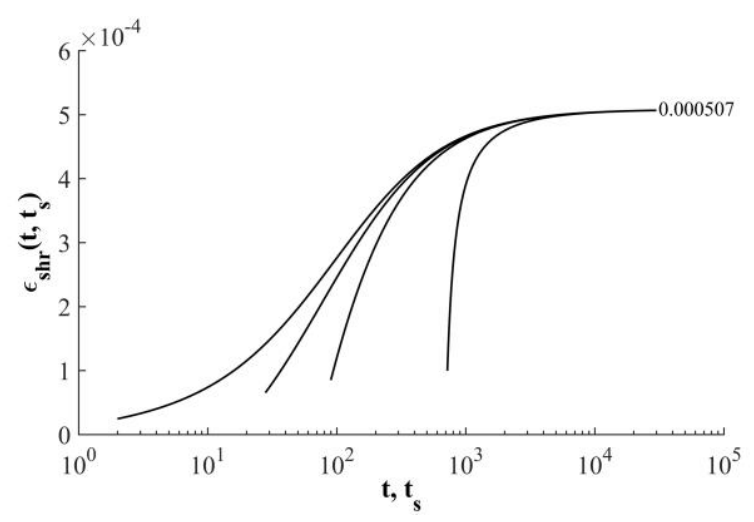

b)

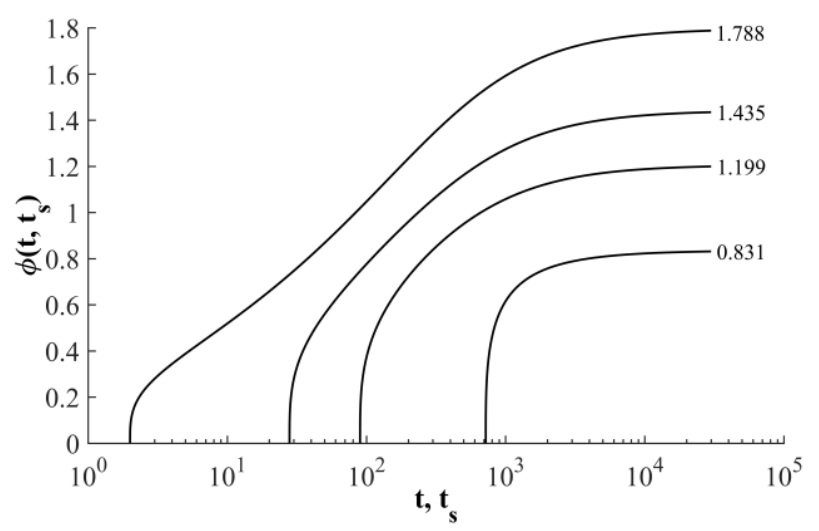

d)

Fig. 1 Evolution of concrete creep and shrinkage strains with time: a) shrinkage strain for compressive strength class of concrete $\mathrm{C} 12 / 15$, b) the same for $\mathrm{C} 50 / 60$; c) creep coefficient for $\mathrm{C} 12 / 15$, d) the same for C50/60

In Fig. 1, the evolution of creep coefficients and free shrinkage strains are demonstrated for the strength classes of concrete of $\mathrm{C} 12 / 15$ and $\mathrm{C} 50 / 60$. The free shrinkage initiation time is assumed to be at $t_{\mathrm{s}}=2,28,90$ and 720 days. The time equals $t_{\mathrm{s}}=2$ can be assumed to model a start of shrinkage strain in the naturally hardening concrete. The initiation of shrinkage strains at 28,90 and 720 can be treated to model the shrinkage in the sealed reinforced concrete specimens. It means that shrinkage starts developing only after removing the sealing.

In Fig. 2, two cases of analysis are presented. In particular, the time-invariant and the time-variable cases of elasticity modulus, for $E_{c}(t) \neq E_{c}\left(t_{0}\right)$ are adopted. These 
cases are selected since the evaluation of partially recoverable instantaneous strains with time mathematically complicate solution of Volterra's integral.

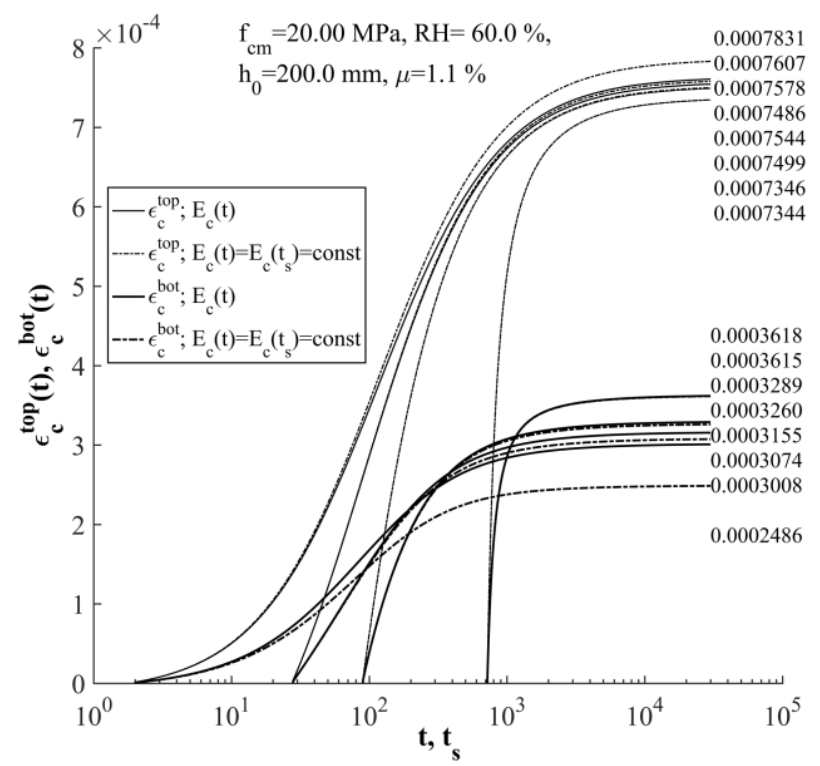

a)

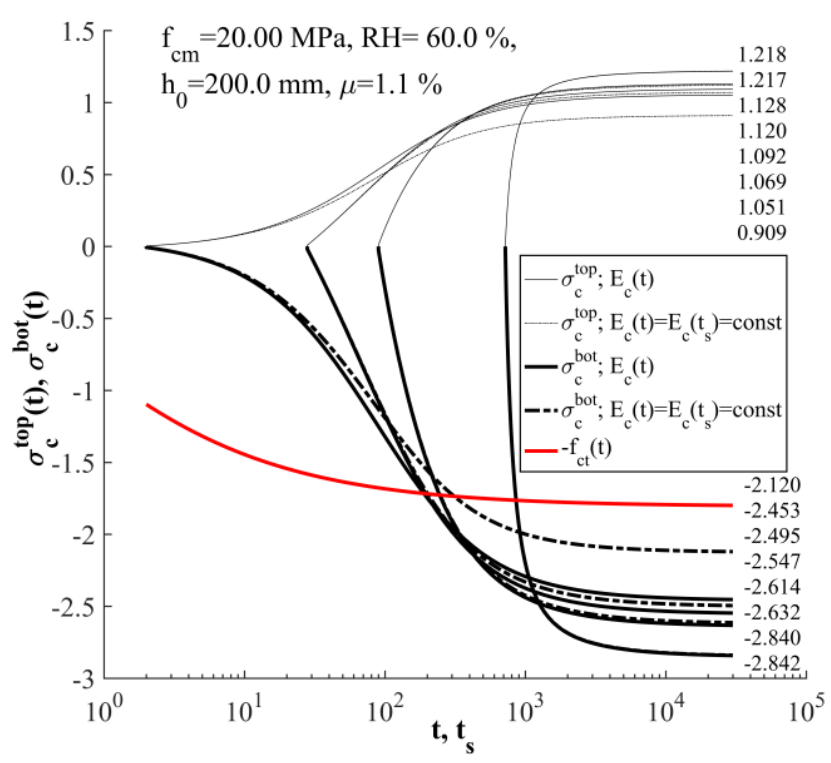

c)

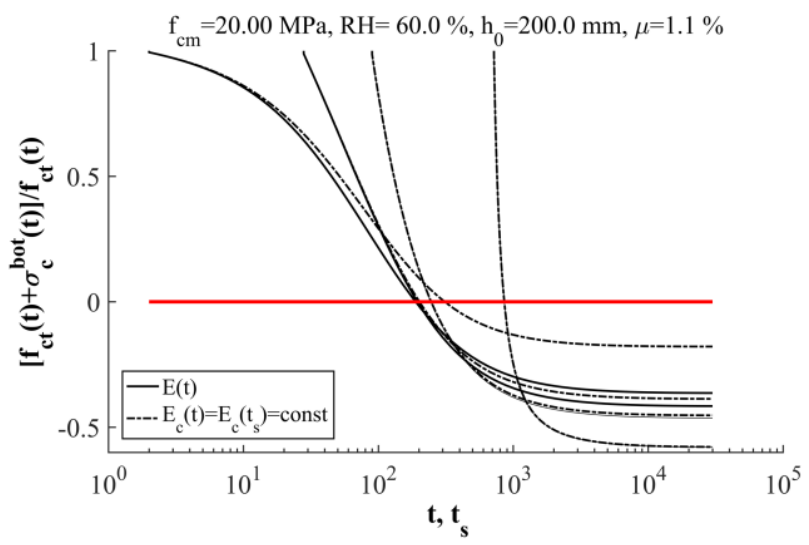

e)
The graphs of concrete strain (Fig. 2, a), predicted at the top and bottom fibre indicate that due to shortening the reinforced concrete cross-section is fully compressed.

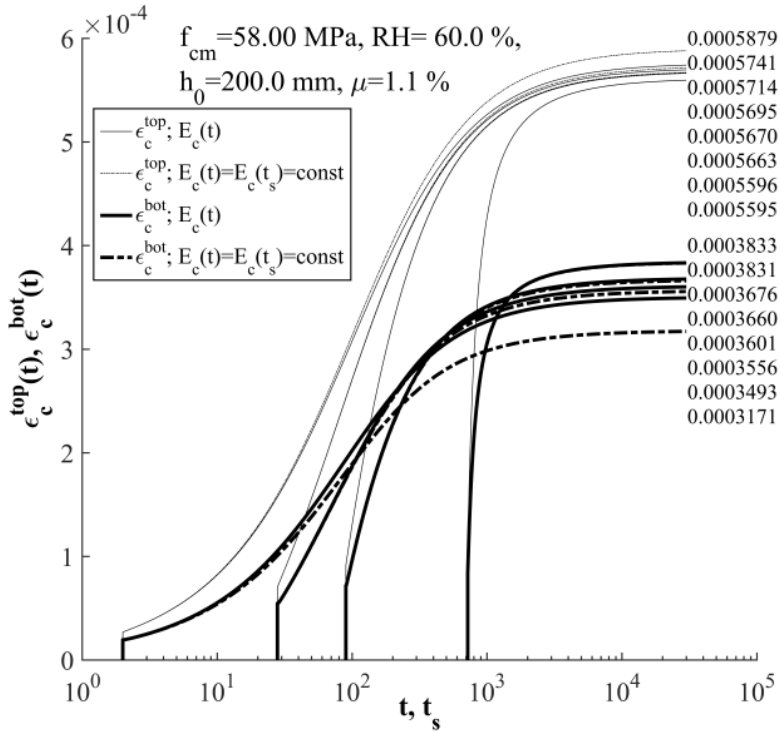

b)

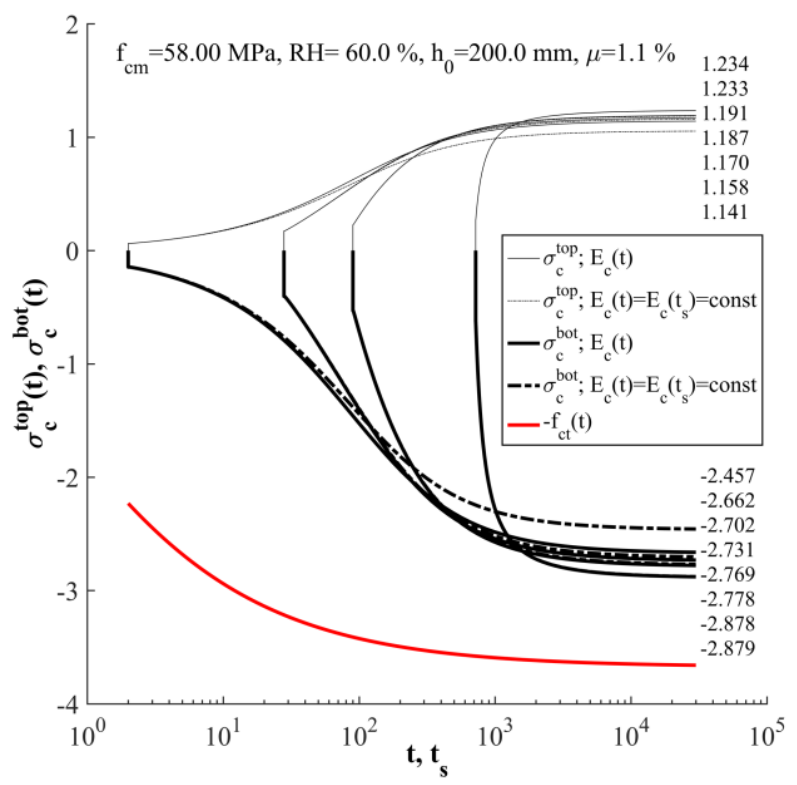

d)

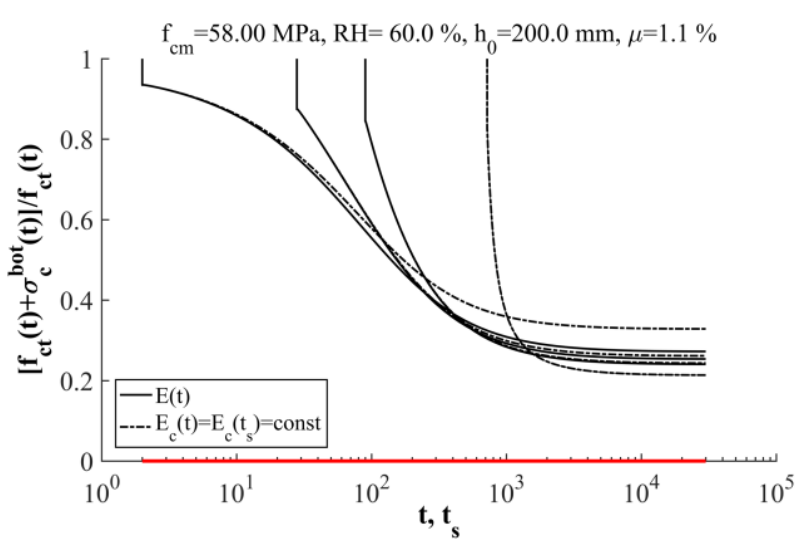

f)

Fig. 2 Stress, strain and strength evolution with time for the cross-section with the reinforcement ratio of $\mu=1.1 \%$ : a, c, e) for strength class $\mathrm{C} 12 / 15 ; \mathrm{b}, \mathrm{d}, \mathrm{f}$ ) for $\mathrm{C} 50 / 60$ 
It can be seen that strain in the top fibre are greater than the concrete strain in the bottom fibre, since at the top fibre of the cross-section there is no reinforcement bars. In this case, at the bottom fibre, presences of reinforcement confine the evolution of free shrinkage strain producing the reduction in the concrete strain in the bottom fibre of the cross-section. Both compressive strain yields neutral axis location beyond outside the cross-section. However, due to self-balancing equilibrium conditions the stress acting in concrete are of different signs. In particular, the tensile stress of concrete is produced in the bottom fibre due to the restrained effect resulted from the reinforcement bars (Fig. 2, b). Meanwhile, the compressive stress evolves with time in the top fibre of the cross-section.

As can be seen, the tensile stress gradually evolves

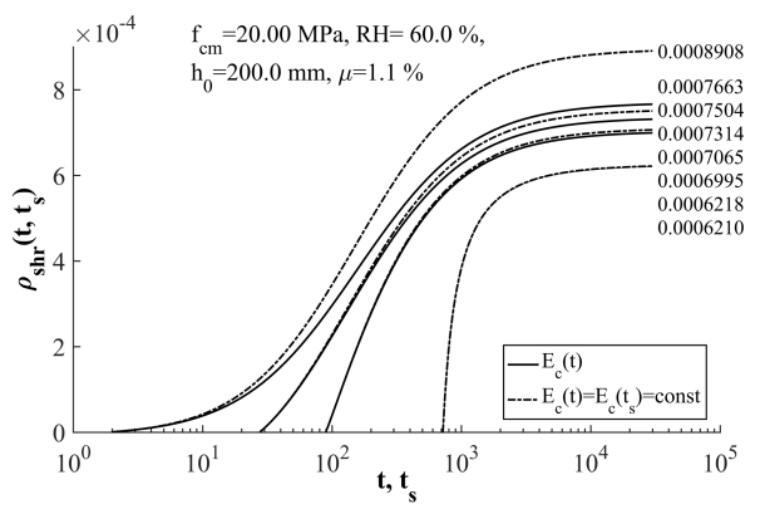

a) with time in accord with development of the shrinkage strain in time. The evolution of tensile stress with time can cause the early age cracking, when this stress reaches the value of tensile strength $f_{c t}(t)$. As can be seen in Fig. 2, c, the first cracks occur starting from 200 days after concreting for concrete with compressive strength class of C12/15. In this case, when the element will be loaded at the age above 200 days, the element will be initially cracked due restrained shrinkage. For high-strength concrete, possessing C50/60 (Fig. 2, d), the cracking resistance is fully satisfied along infinitely long period when the shrinkage strain evolves in time. The effect of tensile strength reduction due to the shrinkage-induced tensile stress in the bottom fibre of the cross-section is demonstrated in Fig. 2, e.

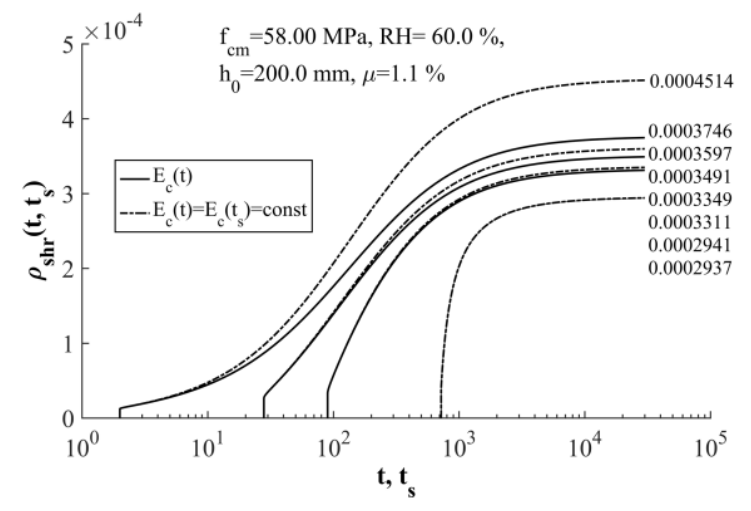

b)

Fig. 3 Evolution of the restrained shrinkage induced curvatures for the cross-section with the reinforcement ratio of $\mu=1.1 \%$ : a) for strength class $\mathrm{C} 12 / 15$; b) for C50/60

A time-dependent evolution of element curvature, for the cases of time-invariant and time-variable modulus of elasticity is presented in Fig. 3. As can be seen in these graphs, the gradual increase in the instantaneous strain with time is very important for proper evaluation of the shrinkage-induced curvature of the element, especially, when the hydration process start to evolve naturally at early age of concrete hardening. Since the increase in the values of modulus of elasticity of concrete with time produces the partially recoverable instantaneous strains, the increase in the element curvature values can reach up to $15 \%$ from those obtained by neglecting the time-evolution modulus of elasticity. This difference is higher for the higher class of concrete. It is clear that there is no difference in curvature values for both cases for old concrete (e.g., when the concrete specimen is sealed by about 100 days and next is exposed to the natural environmental conditions).

In Fig. 4, b and 4, c, it is demonstrated that the cracking resistance can be assessed by introducing the dimensionless factor which subtracts the tensile stress at the bottom fibre of the cross-section from the tensile strength of concrete with dividing by the tensile strength of concrete. When the tensile stress value becomes equal to the tensile strength the first cracks are appeared. So, by accounting for the effect produced by restrained shrinkage, developing in a period prior the element loading, the values of cracking moment of the cross-section can be corrected by multiplying the tensile strength by this factor.

The effect of reinforcement to time-dependent evolution of strain-strain state is demonstrated by selecting by the increased amount of reinforcement up to $\mu=2.3 \%$ (cf.,
Fig. 4). The obtained results (cf., Fig. 2, a, b and Fig. 4, a, b) show that the strain values at the top fibre of the crosssection are higher up to $7 \%$ and lower at the bottom fibres up to $33 \%$ for the case of $\mu=2.3 \%$. The increase of reinforcement ratio values from $\mu=1.1 \%$ up to $\mu=2.3 \%$, for the elements made of concrete class grade $\mathrm{C} 20 / 25$ concrete reduce the time of initiation of first crack from 200 till 100 days. Meanwhile, for the elements with C50/60, the cracks will not appear during the final time considered ( $t \rightarrow \infty$ days), when $\mu=1.1 \%$. For elements with reinforcement ratio $\mu=2.3 \%$ the cracks can appear after 300-400 days. Thereby, due to restrained shrinkage the cracking resistance of the element is highly diminishes with increase in the amount of reinforcement.

A shrinkage-induced evolution of the coefficient of aging is demonstrated in Fig. 5. It is important to note; that coefficient of ageing is strongly dependent on intense evolution of stress. It can be observed the significant drop of values $\chi\left(t, t_{0}\right)$ up to $0.35-0.5$ occurs at first 28-30 days after starting of natural hardening of unsealed reinforced concrete specimens. The evolution of partially irrecoverable instantaneous strains plays a significant rule in the diminishment of $\chi\left(t, t_{0}\right)$ values. The shrinkage-induced values of the coefficient of ageing are very important for explicit prediction of stress and calculation of cracking resistance in short-term analyses [3].

The descending branches of the ageing coefficient are governed by the material ageing, while, for old concrete, the ageing is minimal and $\chi\left(t, t_{0}\right)$, during a short period 


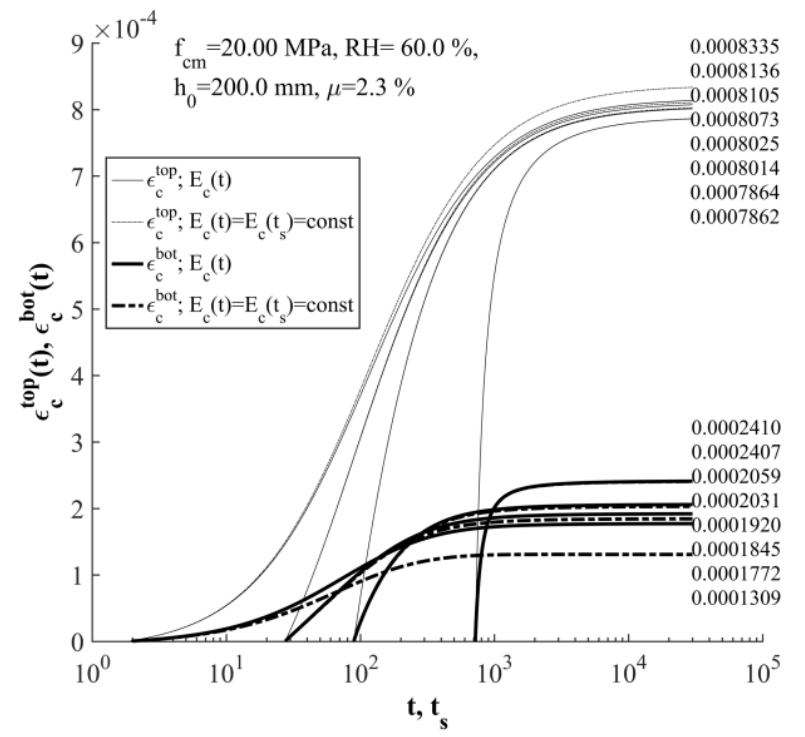

a)

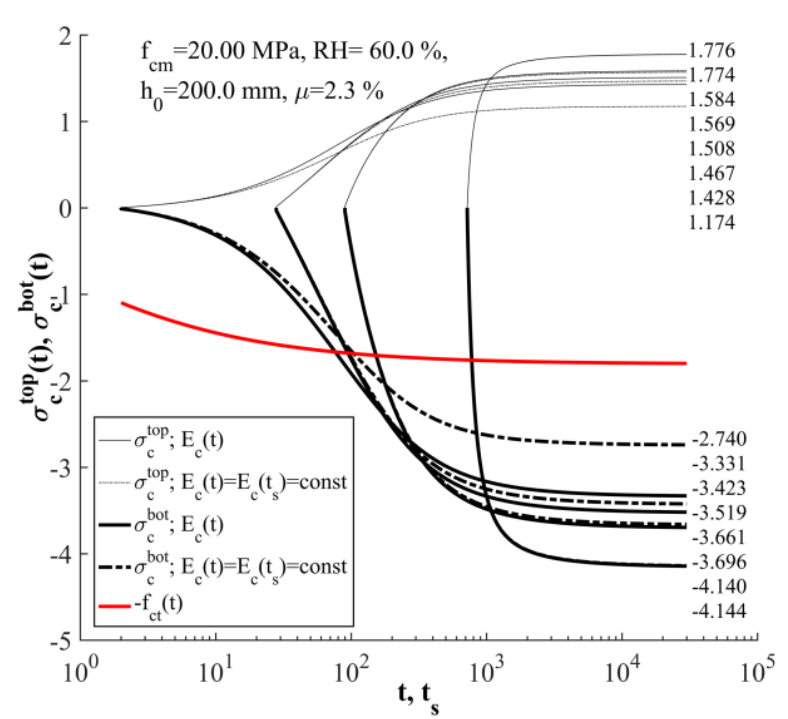

c)

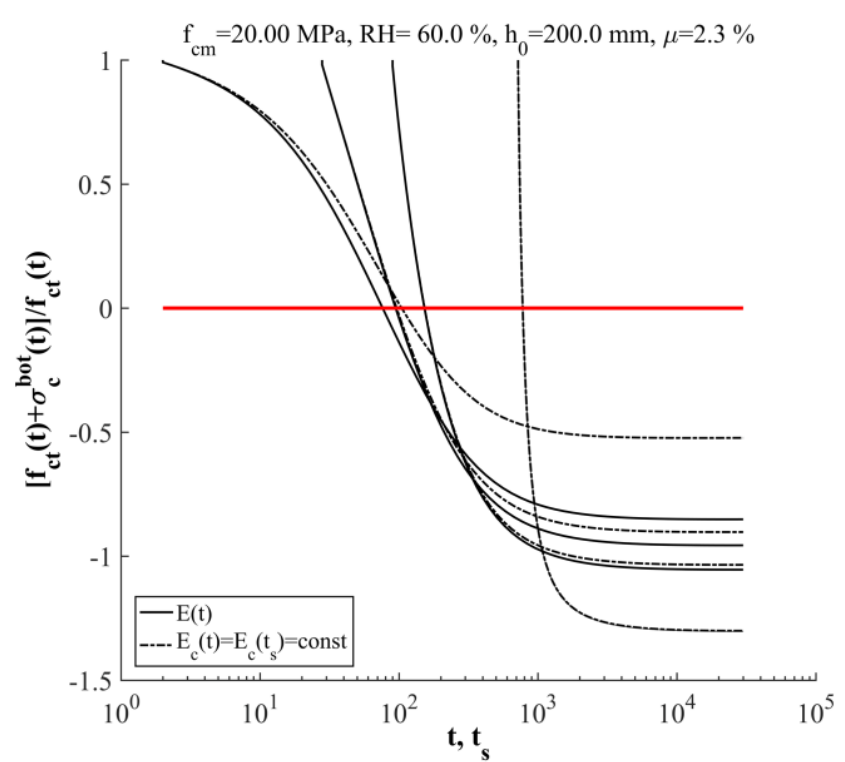

e)

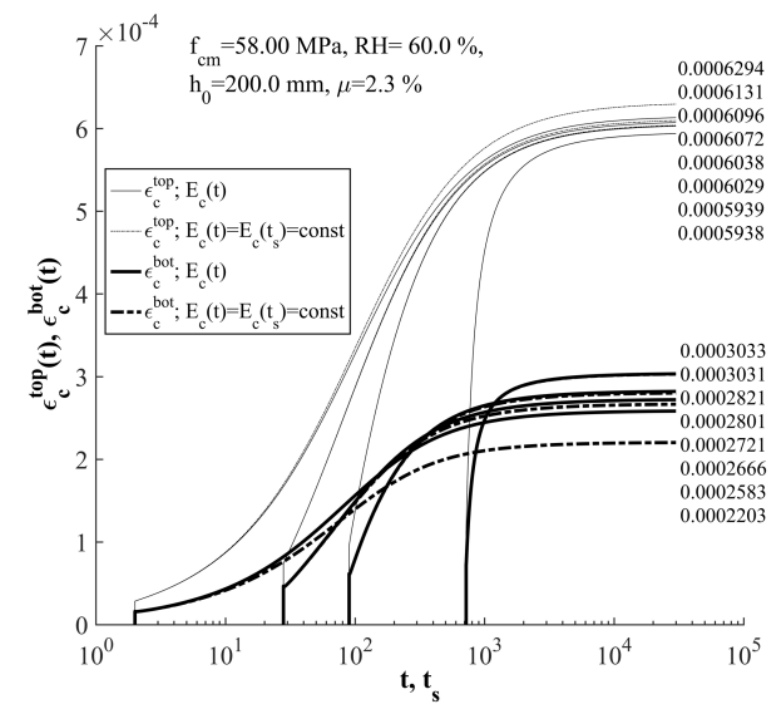

b)

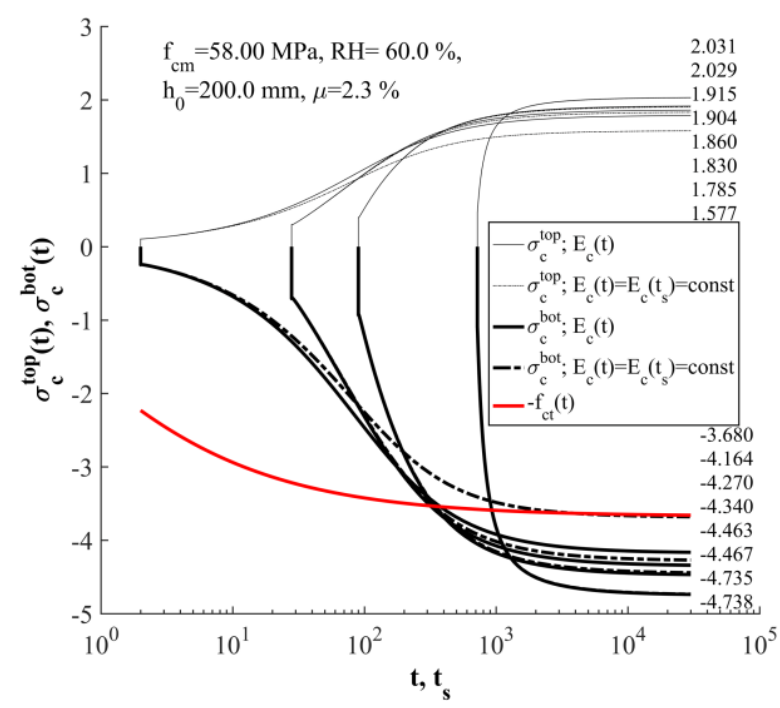

d)

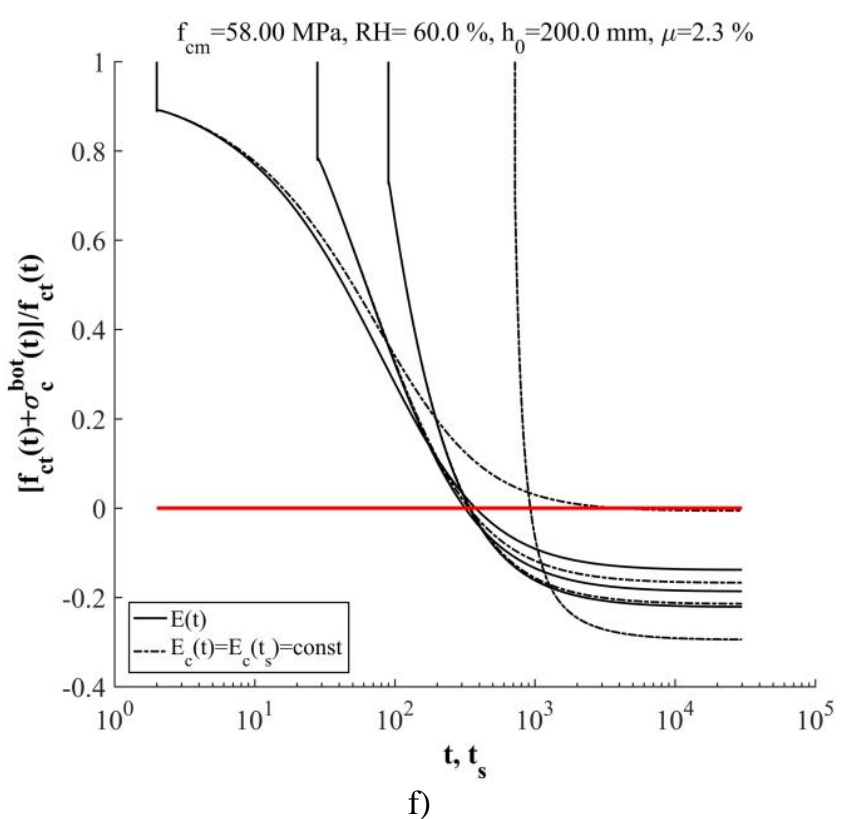

Fig. 4 Stress, strain and strength evolution with time for the cross-section with the reinforcement ratio of $\mu=2.3 \%$ : a,c,e) for strength class $\mathrm{C} 12 / 15$; b, d, f) for C50/60 


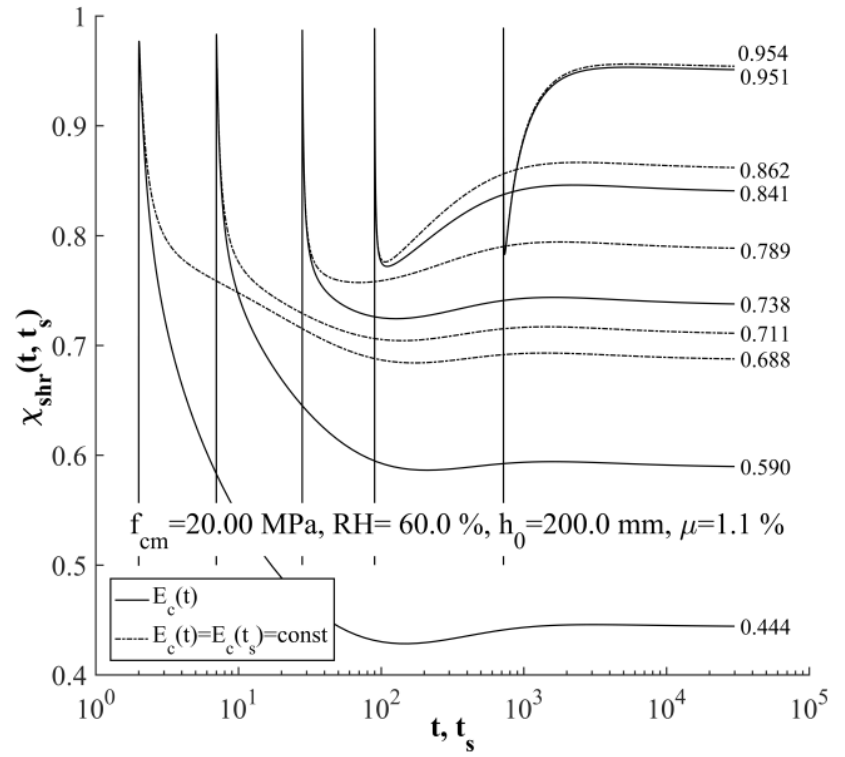

a)

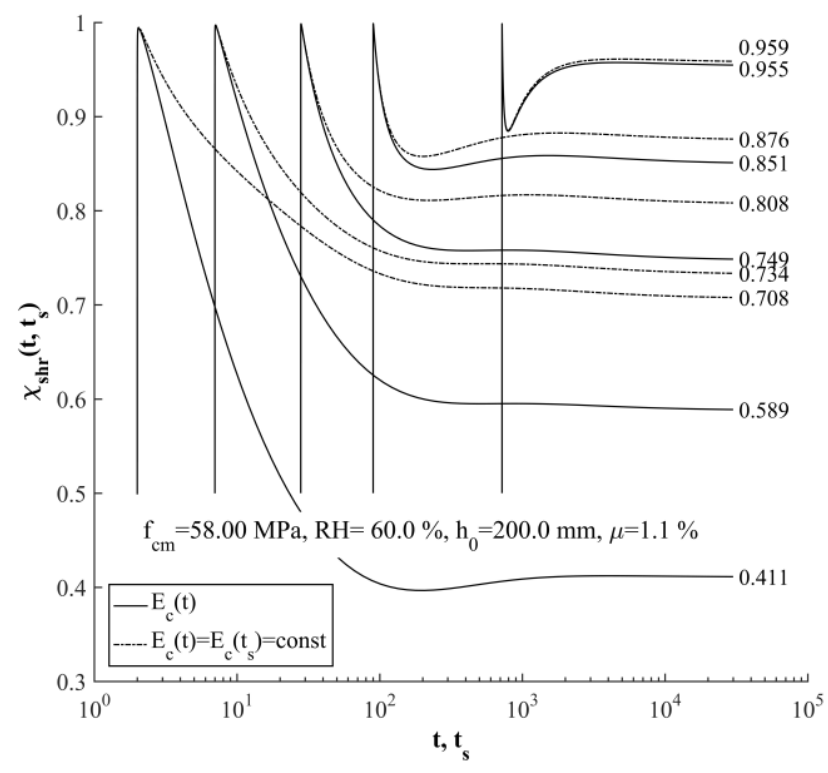

c)

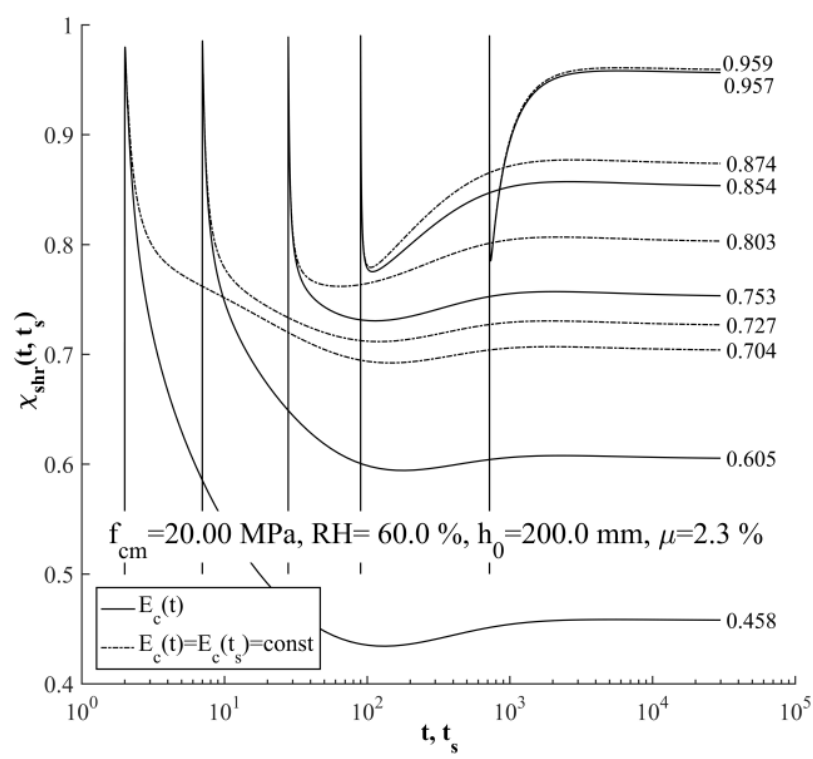

b)

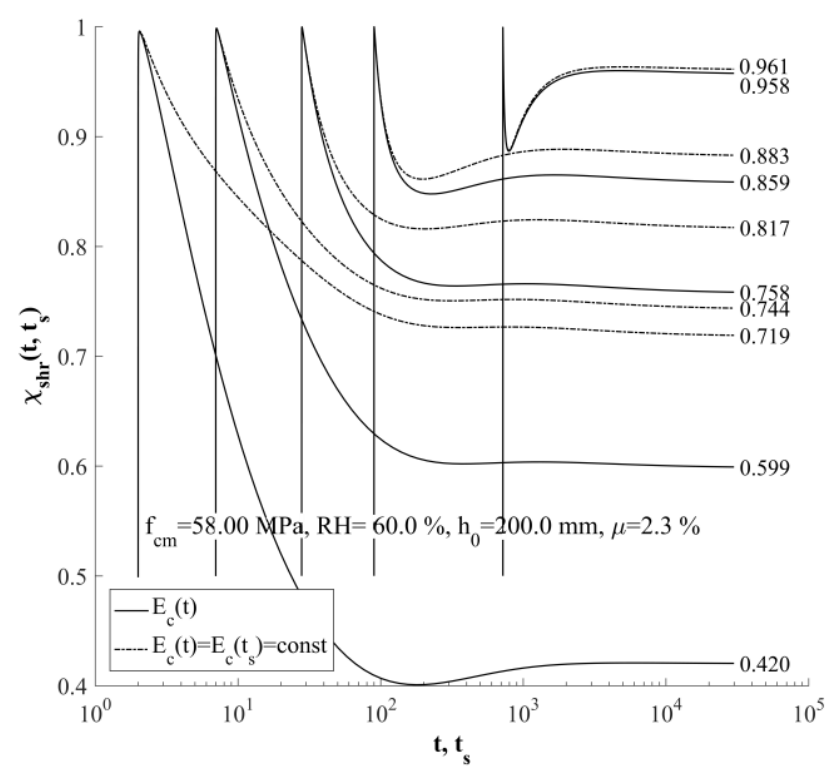

d)

Fig. 5 Coefficients of ageing induced by the restrained shrinkage: a) for $\mu=1.1 \%$ and $\mathrm{C} 12 / 15$; b) for $\mu=2.3 \%$ and $\mathrm{C} 12 / 15$; c) for $\mu=1.1 \%$ and $\mathrm{C} 50 / 60 ; \mathrm{d}$ ) for $\mu=2.3 \%$ and $\mathrm{C} 50 / 60$

tends to 1 . In addition, $\chi\left(t, t_{0}\right)$, for $t>t_{0}+360$, tend to remain constant. The values of $\chi\left(t, t_{0}\right)$ are also important in prestressed concrete structures in calculation of the losses of prestress due to shrinkage.

\section{Concluding remarks}

The time-dependent evolution of stress-strain state induced by the restrained shrinkage developing during concrete hardening can significantly reduce the cracking resistance of reinforced concrete element. The theoretical assessment of this phenomenon is quite complicated, since the stress-strain history should be traced from the inverse analysis of Voltera's integral therm. To this end, the numerical model has been proposed, while the obtained results are adopted in predicting the reduction of the cracking moment of the cross-section and time at which the first crack can appear due restrained shrinkage strain.

On the basis of the obtained results can be also stated that the neglect of partially recoverable instantaneous strains by holding the elastic modulus of concrete as the time-invariant variable can yield significant errors, especially in prediction of the values of the early-age curvatures of the element.

For the exact analytical prediction of the time-dependent stress-strain state induced by the restrained shrinkage, the key parameter is the coefficient of aging which strongly depends on the time-evolution of tensile stress of concrete and modulus of elasticity. The values of this coefficient obtained in the current analysis are suitable for the proper evaluation of the reduction of cracking resistance of the cross-section induced by restrained shrinkage evolving during concrete hardening process. 


\section{References}

1. Balevičius, R. 2010. An average stress strain approach to creep analysis of RC uncracked elements. Mechanics of Time-Depent Materials, 14: 69-89. http//:doi 10.1007/s11043-009-9093-x.

2. Bischoff, P.H.; Scanlon, A. 2007. Effective moment of inertia for calculating deflection of concrete members containing steel reinforcement and FRP reinforcement. ACI Structural Journal 104(1): 68-75.

3. Bischoff, P.H.; Gross. S.P. 2011. Design approach for calculating deflection of FRP reinforced concrete. Journal of composites for construction, 490-499. https:// doi/10.1061/(ASCE)CC.1943-5614.0000195.

4. Bresler, B.; Selna, L. 1964. Analysis of time-dependent behaviour of reinforced concrete structures. Symposium on Creep of Concrete, ACI Special Publication, SP-9(5): 115-128.

5. Gilbert, R.I. 1988. Time effects in concrete structures. Elsevier, Amsterdam, 335 p.

6. Gilbert, R.I.; Ranzi, G. 2010. Time-dependent behaviour of concrete structures. Spon Press, London and New York, $428 \mathrm{p}$.

7. EN 1992-1-1:2004: Part1-1. European Committee for Standardization. Eurocode 2: Design of concrete structures. General rules and rules for buildings. CEN, Brussels.

8. Ghali, A.; Favre, R.; Elbadry, M. 2002. Concrete structures: stresses and deformations. Spon Press, London, $608 \mathrm{p}$.
R. Balevičius, M. Augonis, R. Bistrickaitè, S. Diliūnas

\section{SHRINKAGE EFFECT ON CRACKING RESISTANCE OF FLEXURAL REINFORCED CONCRETE MEMBERS}

S u m m a r y

A time-dependent stress-strain state of reinforced concrete elements induced by gradually developing shrinkage of concrete is analysed. Depending on environmental conditions the early age concrete shrinkage can produce a significant tensile stress in concrete that reduces the cracking resistance of the cross-section. To analyse this phenomenon a numerical model is proposed. For the exact analytical prediction of the time-dependent stress-strain state induced by the restrained shrinkage, the key parameter is the coefficient of aging which is strongly dependent on the time-evolution of tensile stress of concrete and modulus of elasticity. The values of this coefficient obtained in the current analysis are suitable for the proper evaluation of the reduction of cracking resistance of the cross-section induced by restrained shrinkage evolving during concrete hardening process in a period prior to element loading.

Keywords: reinforced concrete, concrete creep, ageing coefficient, time-dependent stress strain state, uncracked cross-sections.

Received April 06, 2017

Accepted February 15, 2018 